\title{
Real Time Implementation of Adaptive Sliding Mode Controller for a Nonlinear System
}

\author{
Srinivasan KANTHALAKSHMI ${ }^{1 *}$, Wincy Pon ANNAL ${ }^{2}$ \\ ${ }^{1}$ PSG College of Technology, Coimbatore, India \\ skl.eee@psgtech.ac.in (*Corresponding author) \\ ${ }^{2}$ Government College of Technology, Coimbatore, India \\ asw@gct.ac.in
}

\begin{abstract}
The objective of this work is to design a controller that may maintain the level of a conical tank which has nonlinear dynamics. Because of the nonlinearity present in the dynamics of the plant, the controller parameters have to be dynamic as well, so that the performance of the system may be enhanced. Hence sliding mode controller which is robust is designed with an adaptive mechanism, so that it could cope up with the varying dynamics of the system. In this paper, three different algorithms were used to study the system behaviour by using simulation. These algorithms were also implemented in real time. When its performance was observed in real time, the adaptive sliding mode controller proved to outperform when compared to reaching law and super twisting algorithm based sliding mode controllers.
\end{abstract}

Keywords: Adaptive control, Nonlinear systems, Pneumatic actuators, Robustness, Sliding mode control.

\section{Introduction}

Automatic control systems find their application in many domains like manufacturing industries, process industries, refineries, nuclear power plants. Knowing the dynamics of the system helps to correct any undesirable situation and aims to provide safety. A control system is designed based on the model behaviour of the process to be controlled. All real world systems are dynamic and nonlinear. Designing controllers for such systems is challenging because the mathematical models used describe only approximately the behaviour of the plant. Also the unmeasured disturbances and the uncertainties present in both internal and external sections of the plant produce adverse effects on the plant which has to be overcome by the designed controller.

Industries use mostly the Proportional Integral Derivative (PID) controllers in their feedback mechanism. This is because of their simple structure and good response when they are finely tuned. Generally fast response and lower offset are the conflicting objectives which require different set of parameters for the PID controller. Also the PID controller gives a response which may be sluggish, aggressive or oscillatory based on its parameters. They are less robust, with poor disturbance rejection capability. They also do not react on change in the process dynamics which happens in the case of nonlinear processes. Robust controllers like sliding mode controller can eliminate such discrepancies.

Sliding mode control (SMC) is a nonlinear control design technique. It ensures robustness in the presence of uncertainties and external disturbances. For certain systems that cannot be smoothly stabilized, sliding mode offers a possible solution in view of the discontinuous nature of the control law as said in (Ahmed et al., 2012). It is accepted as one of the component mechanism for the design of robust controllers when the system is nonlinear and the order of the system is high and when the plant operates under uncertain conditions. The concept of variable structure system and sliding mode as the basic action mechanism is discussed in (Utkin, 1993). Electric drives were used to validate the behaviour of sliding mode control such as order reduction, decoupling design procedure, disturbance rejection, and insensitivity to parameter variation.

Variable structure control with sliding mode was discussed in (Hung, Gao \& Hung, 1993). It gives a brief introduction on the fundamental theory, main results and practical applications of this design. Aspects like invariance, robustness, order reduction and control chattering were conferred on nonlinear systems. Techniques to manage chattering were handled.

In the paper (Moldoveanu, 2014), a new technique using the derivative of the control to eliminate chattering was proposed. This was checked for nonlinear systems with uncertainty.

The applicability of SMC on complex motion steering systems was examined in (Sabanovic, Sabanovic \& Onal, 2005). The applicability of SMC on trajectory tracking and force control has already 
been established in the literature and was focused on the bilateral control (master-slave operation) and the control of multilateral systems in interaction. It shows how SMC framework is suitable for unified formulation of these control problems.

An SMC algorithm can be applied to regulate the position of an electrical machine that is subject to large changes of inertia and load torque. For that purpose, in (Fnaiech et al., 2006), a linear feedback with switched gain approach has been applied. Although it is not often used, it can elevate the chattering effect. The proposed algorithm was applied to a three phase induction machine. To eliminate the reaching phase, a time variant switch surface was introduced into the algorithm.

A variable controller without the requirement of reaching movement was proposed in (Pan et al., 2000). The system control inputs were switched among a set of feedback control laws to stabilize the control system. It does not matter whether a sliding mode takes place or not. Each feedback control law is easily defined because it uses only one state variable with a gain of fixed magnitude but changing sign. The proposed variable structure control was quadratically stable.

Biotechnological reactors control was targeted in (Selisteanu, Petre \& Rasvan, 2007). The stability of this nonlinear process was ensured by a dynamic sliding mode-based feedback strategy. Then the paper extends this study to include the adaptive SMC strategy that was derived for the class of nonlinear systems. The adaptive SMC was designed by direct parameterized method to manage the uncertainties of the bio-processes.

A new method for linearized systems with uncertainties using sliding mode control with fuzzy tuning was discussed in (Ha, Negnivitsky, $\&$ Man, 1996). The control signal consists of three components: the equivalent control for assigning the sliding eigenvalues of the nominal closed loop system, the robust control for driving the representative point in the sliding hyperplane in the reaching phase, and the fuzzy tuned control for accelerating the reaching time and reducing the influence of uncertainty. Chattering was also taken into account. Two mass servo systems were used for the validation.

A single input single output, linear, time invariant system driven by a certain class of bounded errors, nonlinearities and disturbances was considered and a stable and robust sliding mode control was designed. The results were developed using inverse models in (Varghese, Fernandez \& Hedrick, 1988). Conventional sliding mode control systems were dealt in (Feng, Ha \& Yu, 2014). Singularity in terminal sliding mode control system and the chattering in both the conventional linear sliding mode and the terminal sliding mode control systems are the main problem faced in SMC. A full order terminal sliding mode control which could eliminate chattering was proposed here. The control similarities were avoided as the derivative of terms with fractional powers do not appear in the control law. The system acts as a desirable full order dynamics instead of a desirable reduced order dynamics. This allows both the chattering and singularity problems to be solved.

Nonlinear system synthesis was performed in (Setiawan et al., 1997) using sliding mode method. The problem of controlling the input to bring the system state to equilibrium was solved by choosing a manifold or certain area that once the system state enters, it will slide to equilibrium point. By defining an area of a certain surface, the input control for both linear and nonlinear systems could be determined. The chattering effect caused forcing the system to enter the area of sliding surface was the major problem in this method. A controller was developed using a new method for sliding mode control in order to produce or to determine adjustment input control by removing or smoothing chattering effect without disturbing the performance. Replacing the sign function with tan inverse function, the deletion or smoothing of chattering effect was analysed. It was performed by ordering the parameters of the system and by considering the aspects of stability and the system uncertainties.

The problems of the accurate assessment of chattering were addressed in (Young, Utkin \& Zgune, 1999), which paved the way for carrying out the sliding mode control design and which became the starting point for future sliding mode control research.

A new algorithm for discrete time sliding mode control with periodic output feedback technique was explained in (Janardhanan \& Bandyopadhyay, 2005). It uses only the output sample for designing

http://www.sic.ici.ro 
the controller. A similar work was reported in (Dorel, \& Levant A., 2008).

Discrete time integral sliding mode control was implemented on an induction motor in (Chihi et al., 2017). In this paper, an integral switching surface was developed so as to decrease the settling time and steady state error of the system.

A new method that yields a faster response time was proposed in (Temel \& Ashrafiuon, 2012). Here, the control signal was derived from a manifold consisting of a sliding surface and its derivative. It was reported with lower error and minimal control effort.

The power of sliding mode control resides in its robustness. The techniques of switching between two states used was not delicate to parameter variations. Also, the sliding mode control would be reached in a finite time as the control was not continuous. A hypersurface was selected on the manifold along with the feedback gains so that the desired behaviour of the system was attained. This can be very much employed in applications like aircraft movement control where robustness is much required as it was explained in (Srinivasan, Gandhi \& Venugopal, 2014).

The competence of the algorithm is checked in both simulation and real time implementation on a non-interacting conical tank level system which shows dynamic nonlinearity. The adjustment of the controller in real time based on the system dynamics could be done using adaptive controllers. SMC could perform better when adaptation is incorporated.

In this paper, the liquid level control in a noninteracting conical tank is performed using adaptive SMC and the results along with the significance of the adaptation mechanism is reported. The paper is organised as follows: Section 2 presents the modelling of the system, section 3 describes the system under study, section 4 explains the controller design, section 5 produces the results and section 6 gives the conclusion of the paper.

\section{Modelling of the System}

Mathematical modelling of the process gives no information about the transport delays, and disturbances. Also it requires a sound knowledge on the dynamics of the components involved in the flow line of the process. Any mismatch in modelling may lead to performance degradation of the designed controller. So experimental modelling is performed which overcomes the shortfalls faced by mathematical modelling.

To obtain the system model experimentally, the flow of liquid to the tank was permitted and the system was allowed to settle. Then a step change in flow was made while maintaining the outlet valve positions unchanged and the rise in level of liquid was observed until the system reached the steady state. A response curve was plotted from which slope of the curve was calculated. From the change in input and output details, the gain of the system was calculated. Using these data in process reaction curve method, the system was modelled as a first order system with dead time.

\section{System Description}

The experimental setup used for the proposed work shown in Figure 1 consists of a sump from which water is pumped through a control valve to the tank. The outlet of the tank is kept constant by a hand valve. This valve also helps to introduce disturbance in the system by changing the position of the control valve. The inflow of the tank can be changed by varying the lifting of the stem in the control valve. The stem of the valve is actuated by a pneumatic signal proportional to the control signal received from the controller. The abrupt and continuous movement of the stem to vary the inflow of the tank so as to maintain the level in the tank causes damage to the pneumatic actuator, which is generally not considered in the design of controller. This problem is well addressed by adaptive SMC, which reduces the chattering of the control valve, leading to smooth variations in stem movement, thus increasing the life of the pneumatic actuator.

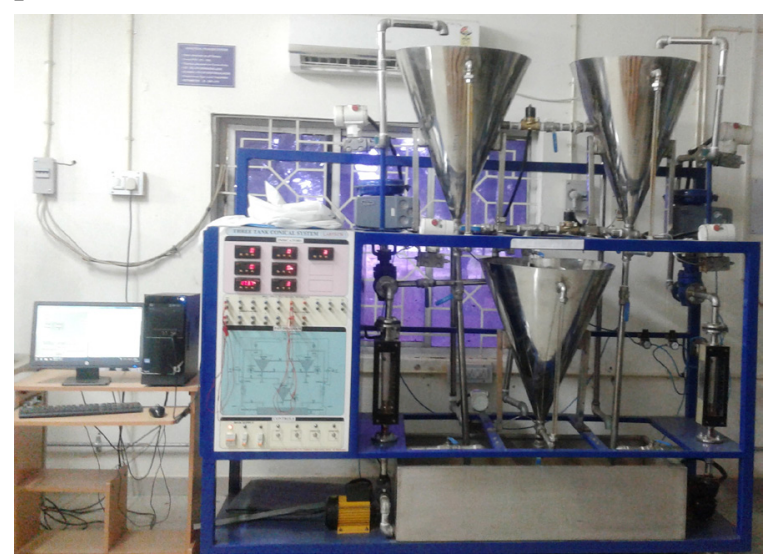

Figure 1. Experimental setup 


\section{Controller Design}

Considering the nonlinear plant as

$\theta(t)=-f(\theta, t)+b u(t)$

where $f(\theta, t)$ and $b$ are the system parameters, and $\mathrm{u}(\mathrm{t})$ is the control variable, the control algorithms are described.

\subsection{Sliding Mode Controller Based on Reaching Law}

Sliding mode controller based on reaching law is determined using two parts: reaching phase and sliding phase. The reaching phase drives the system to maintain a stable manifold. The system is made to slide to equilibrium in the sliding phase. When the states of the system are far away from the switching manifold, the reaching speed is increased. However it reduces the rate at which the state is reached when it is near the manifold, but the chattering is weakened by moving the system state close to the sliding phase plane. It involves the design of the sliding surface, and control input to make the switching manifold $\mathrm{s}=0$. This controller enforces the switching variable near the switching manifold $\mathrm{s}$ to a constant rate $\varepsilon$. The sliding mode function is

$$
s(t)=c e(t)+\dot{e}(t)
$$

where $\mathrm{e}$ is the error and $\mathrm{c}$ is a constant selected such that it satisfies the Hurwitz condition, $\mathrm{c}>0$.

Therefore,

$$
\dot{s}(t)=c\left(\dot{\theta}_{d}-\dot{\theta}\right)+\left(\ddot{\theta}_{d}+f(\theta, t)-b u(t)\right)
$$

where $\theta_{\mathrm{d}}$ is the set point.

According to the constant rate reaching law,

$$
\dot{s}=-\varepsilon \operatorname{sgn}(s), \varepsilon>0
$$

From (3) and (4), the sliding mode controller equation becomes,

$$
u(t)=\frac{1}{b}\left(\varepsilon \operatorname{sgn}(s)+c\left(\dot{\theta}_{d}-\dot{\theta}\right)+\ddot{\theta}_{d}+f(\theta, t)\right.
$$

\subsection{Sliding Mode Controller Based on Super Twisting Algorithm}

The dangerous chattering effect prevents the conventional sliding mode control from being extensively used in practice. While reducing the chattering, the modified sliding mode control with boundary layer fails to track the set point and loses its robustness. The second order sliding mode control is a possible way to reduce the chattering effect without affecting the tracking performance and robustness. Second derivative of the sliding variable is used in second order sliding mode controller. This will retain the robustness of the controller while reducing the chattering. But the major problem is the requirement of information regarding the first derivative of the sliding variable. This is overcome by the super twisting sliding mode control scheme.

If $s=0$ was the selected sliding manifold, then the objective of the control would be to apply a second order sliding mode on the sliding manifold $\mathrm{s}=0$. The derivative of $\mathrm{u}(\mathrm{t})$ is the auxiliary control variable which drives the sliding variable to zero and avoids chattering when maintained continuously. Taking the first and second derivative of the sliding variable,

$$
\begin{aligned}
& \dot{s}=\frac{\partial}{\partial t} s+\frac{\partial}{\partial \theta} s[f(\theta, t)+b u(t)] \\
& \ddot{s}=\varphi_{A}(\theta, t, u)+\gamma_{A}(\theta, t, \dot{u}(t))
\end{aligned}
$$

where,

$$
\gamma_{A}(\theta, t)=\frac{\partial}{\partial \theta} s(\theta, t) b
$$

From the above equations, it is clear that the control input $\mathrm{u}(\mathrm{t})$ affects the term $\varphi_{A}(\theta, t, u)$ and its first derivative $\dot{u}(t)$ satisfies the control objective of steering s and $\dot{s}$ to zero. In this case, the auxiliary control variable $\dot{u}(t)$ affects the dynamics of $\ddot{s}$. As the control signal is continuous, the chattering effect is reduced. To reduce chattering without degrading the system performance for the relative degree one systems, super twisting controller mode is a good alternative for the conventional SMC. The aim of control is to force the system trajectory to reach the sliding manifold $s=\dot{s}=0$ in definite time. Solving these equations, super twisting sliding mode controller is given by

$u=-c_{1} \sqrt{|s|} \operatorname{sgn}(s)-c_{2} \operatorname{sgn}(s)$

where

$$
\left\{\begin{array}{l}
c_{2}>\frac{\phi}{\Gamma_{\text {min }}} \\
c_{1}^{2} \geq \frac{4 \phi \Gamma_{\text {max }}\left(c_{2}+\phi\right)}{\Gamma_{\min }^{2} \Gamma_{\min }\left(c_{2}-\phi\right)}
\end{array}\right.
$$

where $\phi, \Gamma_{\min }, \Gamma_{\max }$ are all positive constants. 
If the conditions given in (10) are satisfied, the control given by (9) drives the sliding variable and its derivatives to zero in a finite time (Perruquetti \& Barbot, 2002.)

\subsection{Adaptive Sliding Mode Controller Based on Fuzzy Switching}

Designers who use fuzzy logic to develop sophisticated control systems are finding support from adaptive technology. By including adaptive control in their arrangements, they are able to design systems that can accommodate to environmental changes. An adaptive control system adjusts its response surface to varying signal parameters. An adaptive system modifies the characteristics and the values of the rules in its knowledge repository. The attributes of fuzzy region created for this variable solution are stored in a time or process phased buffer where they can be accessed. Adaptive systems usually work like back propagation neural networks, by examining a solution with a target result (Wang, 1993).

The adaptive fuzzy sliding mode control algorithm is proposed for a class of continuous-time unknown nonlinear systems. The nonlinearities which are unknown are approximated using a set of fuzzy rules in the fuzzy system, whose parameters are adjusted based on the adaptive laws. This aids in controlling the output of the nonlinear system to drag a given trajectory. The chattering action is attenuated and robust performance can be ensured. This method was also used to control the speed of a brushless DC motor in (Kumar \& Kumar, 2010).

Using the adaptive fuzzy control method, the switching in the sliding mode controller is approximated and continued. Therefore the chattering phenomenon can be sufficiently reduced. Using the sliding mode function defined in (2), the sliding mode controller is designed as,

$u(t)=\frac{1}{b}\left(u_{s w}+c\left(\dot{\theta}_{d}-\dot{\theta}\right)+\ddot{\theta}_{d}+f(\theta, t)\right)$

where $u_{s w}=\varepsilon \operatorname{sgn}(s)$

From (3),

$$
s(t)=c\left(\dot{\theta}_{d}-\dot{\theta}\right)+\left(\ddot{\theta}_{d}+f(\theta, t)-b u(t)\right)
$$

Also $s=E(t)-\varepsilon \operatorname{sgn}(s)$

$$
s \dot{s}=E(t) s-\varepsilon|s| \leq 0
$$

When $\mathrm{E}$, the unknown disturbance is relatively large, the switching term $\varepsilon$ in (11) can also be large, thus resulting in serious chattering. Fuzzy system is used to approximate $\varepsilon \operatorname{sgn}(\mathrm{s})$. Therefore the switching signal is weakened and the chattering can be reduced.

Using product deduce, single-valued fuzzy and center average fuzzy, the fuzzy output is $\vec{h}$. From (11), the controller is written as,

$$
\begin{aligned}
& u(t)=\frac{1}{b}\left(\vec{h}(s)+c\left(\dot{\theta}_{d}-\dot{\theta}\right)+\ddot{\theta}_{d}+f(\theta, t)\right) \\
& \vec{h}\left(s \mid \theta_{h}\right)=\theta_{h}^{T} \phi(s)
\end{aligned}
$$

where $\vec{h}\left(s \mid \theta_{h}\right)$ is the fuzzy output of universal approximation, $\phi(s)$ is the fuzzy vector and vector $\theta_{h}^{T}$ varies according to the adaptive rule. The ideal $\vec{h}\left(s \mid \theta_{h}\right)$ is

$\vec{h}\left(s \mid \theta_{h}^{*}\right)=\varepsilon \operatorname{sgn}(s)$

The adaptive rule is

$\dot{\theta}_{h}=\gamma s \phi(s)$

where $\gamma>0$.

\section{Results}

\subsection{Open Loop Response}

The open loop response of the system for a step change in the pneumatic actuator signal was obtained as illustrated in Figure 2.

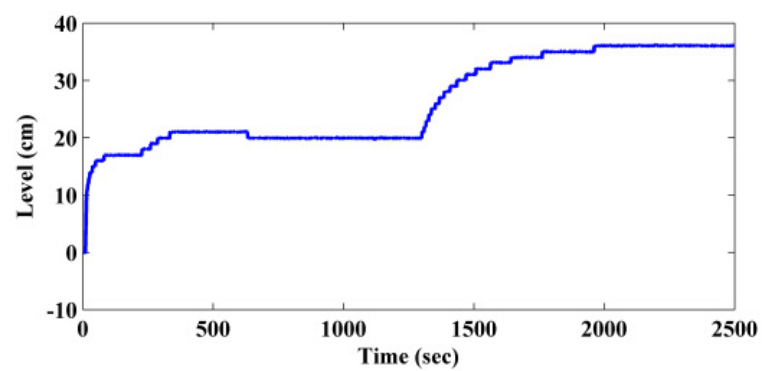

Figure 2. Open loop response of the system 
The model for the operating region $(20-40) \mathrm{cm}$ was obtained using the process reaction curve method and has been used for simulating all of the algorithms. The state space equation was obtained for the system from the open loop response as

$$
\begin{aligned}
& {\left[\begin{array}{l}
\dot{\mathrm{x}}_{1} \\
\dot{\mathrm{x}}_{2}
\end{array}\right]=\left[\begin{array}{cc}
-0.1283 & -0.0004 \\
1 & 0
\end{array}\right]\left[\begin{array}{l}
\mathrm{x}_{1} \\
\mathrm{x}_{2}
\end{array}\right]+\left[\begin{array}{l}
1 \\
0
\end{array}\right] \mathrm{u}} \\
& \mathrm{y}=\left[\begin{array}{ll}
-0.0021 & 0.0003
\end{array}\right]\left[\begin{array}{l}
\mathrm{x}_{1} \\
\mathrm{x}_{2}
\end{array}\right]
\end{aligned}
$$

Using (17) and (18), all the algorithms were simulated.

\subsection{Simulation Results}

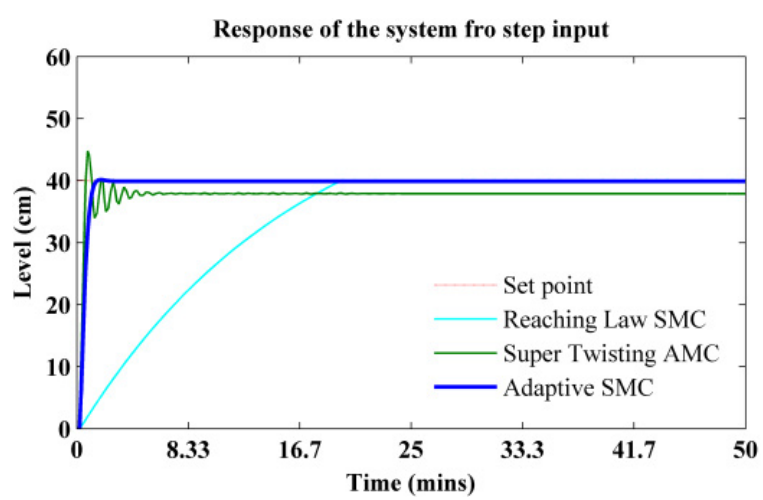

Figure 3. System response for a setpoint of $40 \mathrm{~cm}$

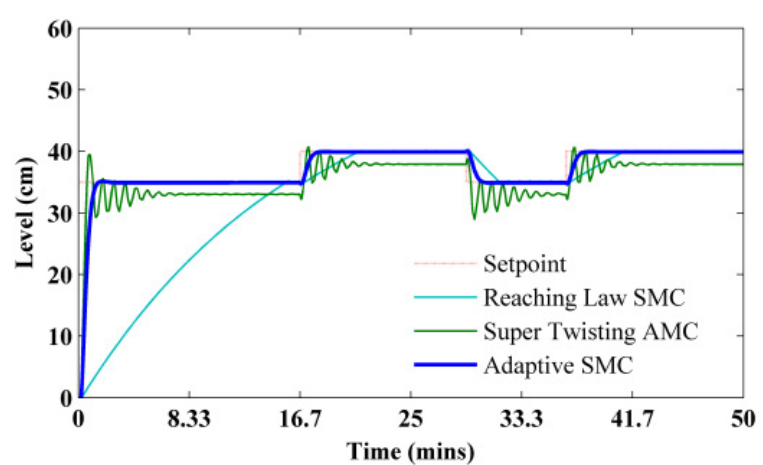

Figure 4. System response for a setpoint tracking

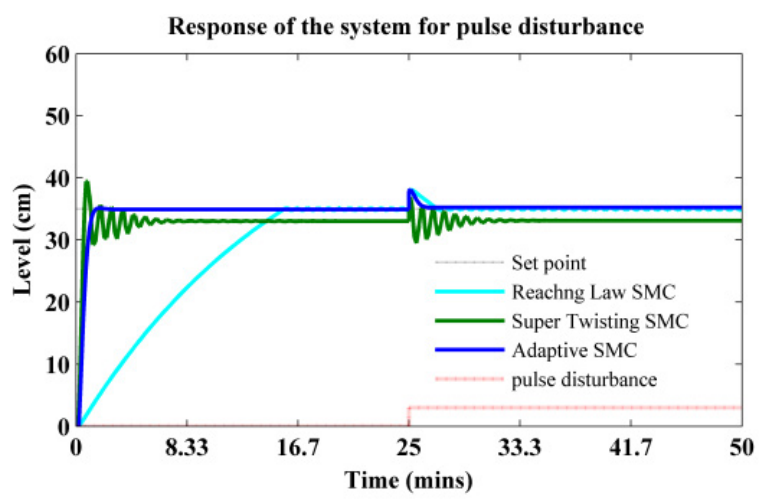

Figure 5. System response subject to disturbance

By comparing the three algorithms for a step change in the input, from Figure 3 it becomes obvious that the adaptive SMC seems to settle much quicker than the other two algorithms. The offset is minimized and the root mean square error (RSME) is also lower. From Figure 4, it is clear that the adaptive SMC tracks the changes in set point much better than the other two algorithms. Figure 5 and Figure 6 also prove that the disturbance rejection is much higher when compared with others.

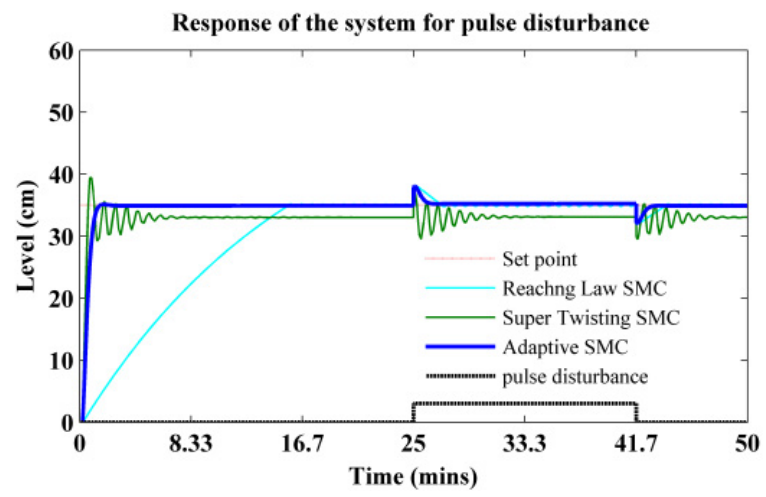

Figure 6. System response subject to pulse disturbance

Adaptive SMC is found to be better even when a disturbance is experienced for a short interval of time which is shown in Figure 6 as a pulse disturbance. The comparison of these results are numerically tabulated in Table 1 as well.

Table 1. Comparison of Responses in Simulation

\begin{tabular}{|c|c|c|c|c|c|c|}
\hline Controller & $\begin{array}{c}\text { Rise } \\
\text { time } \\
(\mathrm{min})\end{array}$ & $\begin{array}{c}\text { Settling } \\
\text { time } \\
(\mathrm{min})\end{array}$ & $\begin{array}{c}\text { Delay } \\
\text { time } \\
(\mathrm{min})\end{array}$ & $\begin{array}{c}\text { Over } \\
\text { shoot } \\
(\%)\end{array}$ & RMSE & $\begin{array}{c}\text { Offset } \\
(\%)\end{array}$ \\
\hline $\begin{array}{c}\text { Reaching } \\
\text { Law }\end{array}$ & 14.95 & 19.842 & 7.288 & - & 4.656 & 0.225 \\
\hline $\begin{array}{c}\text { Super } \\
\text { Twisting } \\
\text { SMC }\end{array}$ & 0.384 & 7.995 & 0.422 & 18.35 & 4.103 & 5.4 \\
\hline $\begin{array}{c}\text { Adaptive } \\
\text { SMC }\end{array}$ & 0.73 & 2.665 & 0.56 & - & 3.437 & 0.15 \\
\hline
\end{tabular}

\subsection{Real Time Results}

The real time response in Figure 7 shows that the offset is minimum when adaptive SMC is used.

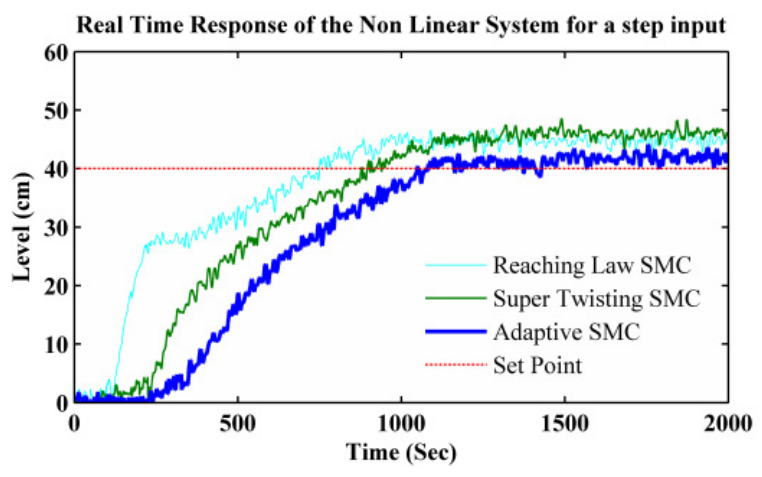

Figure 7. Response of the system in real time 


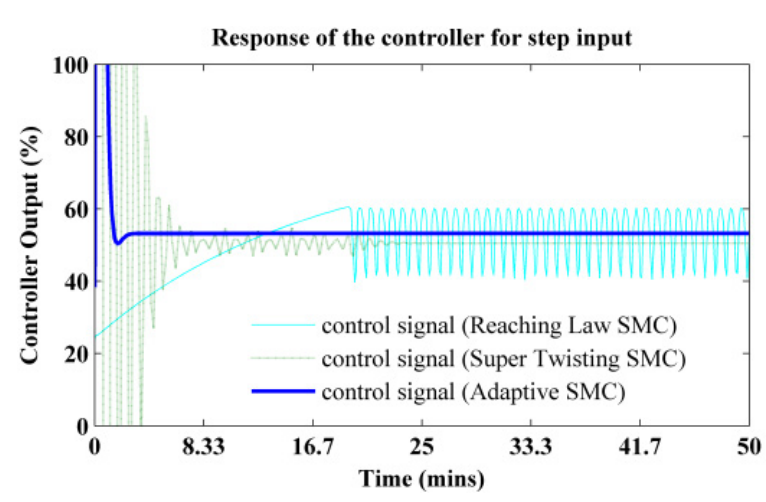

Figure 8. Controller response

Though rise time and delay time increase, the settling time is almost the same in all three controllers. But the chattering experienced when using adaptive SMC is minimal as in Figure 8. The reduction in chattering helps to increase the lifetime of the actuator. In this aspect, the advantage of adaptive SMC is far superior to the other two algorithms. Table 2 reveals the results of all the algorithms when implemented in real time.

Table 2. Comparison of Responses in Real Time

\begin{tabular}{|c|c|c|c|c|c|c|}
\hline Controller & $\begin{array}{c}\text { Rise } \\
\text { time } \\
(\mathrm{min})\end{array}$ & $\begin{array}{c}\text { Settling } \\
\text { time } \\
(\mathrm{min})\end{array}$ & $\begin{array}{c}\text { Delay } \\
\text { time } \\
(\mathrm{min})\end{array}$ & $\begin{array}{c}\text { Over } \\
\text { shoot } \\
(\%)\end{array}$ & RMSE & $\begin{array}{c}\text { Offset } \\
(\%)\end{array}$ \\
\hline $\begin{array}{c}\text { Reaching } \\
\text { Law }\end{array}$ & 8.33 & 20.2 & 3.1 & - & 12.404 & 18.25 \\
\hline $\begin{array}{c}\text { Super } \\
\text { Twisting } \\
\text { SMC }\end{array}$ & 9.4 & 20 & 6.18 & - & 16.625 & 9.75 \\
\hline $\begin{array}{c}\text { Adaptive } \\
\text { SMC }\end{array}$ & 11.67 & 19.21 & 8.75 & - & 19 & 3.25 \\
\hline
\end{tabular}

\section{Conclusion}

The basic principle and design procedures of the three sliding mode controller algorithms

\section{REFERENCES}

1. Ahmed, A. A, Ahmad, R. B, Alobaidi, S. A., Yahya, A., Hassnawi, L. A., Ali, Z. G. \& Tahir, H. H. (2012). Sliding mode simulation and robustness controller, Journal of Next Generation Information Technology, 3(2) 19-27.

2. Chihi, A., Azza, H. B., Jemli, M. \& Sellami, A. (2017). Nonlinear discrete-time integral sliding mode control of an induction motor: Real time implementation, Studies in Informatics and Control, 26(1), 23-32. doi:10.24846/v26i1y201703

3. Dorel, L. \& Levant, A. (2008). On chattering free sliding mode control. In Proceedings of the 47th IEEE conference on Decision and Control (pp. 2196-2201). were learnt and implemented. While comparing the simulated results of the studied algorithms, the adaptive fuzzy-based sliding mode control algorithm proved to be the best suited for multiple set point tracking of the considered nonlinear system. The response showed perfect disturbance rejection capability. The major hindrance which prevailed in all the learned algorithms, was the continuous appearance of chattering in the output response. But, the design of adaptive fuzzy-based sliding mode controller involves the removal of the parameters that caused the chattering. Hence it helped to completely eliminate the chattering in the response. Thus it is proved that adaptive fuzzy control method is best suited for real time controller implementation.

While implementing the learnt controller algorithms in real time non-interacting conical tank system, the responses differed in the view of setpoint tracking. The response of the system showed major offsets for reaching law and super twisting algorithm in contradiction to simulation results which may be due to modelling errors. When applying the adaptive fuzzybased sliding mode controller algorithm on the process, the results showed satisfactory control implementation over the process. Thus when comparing the simulation and real time results, it could be concluded that the adaptive sliding mode controller based on fuzzy switching was the most appropriate controller for the nonlinear system under study.
4. Feng, Y., Ha, F. \& Yu, X. (2014). Chattering free full-order sliding-mode control, Automatica, 50(4), 1310-1314.

5. Fnaiech, M. A, Betin, F., Fnaiech, F. \& Capolino, G. A. (2006). Sliding mode control for dual three-phase induction motor drives. In IEEE International Symposium on Industrial Electronics (pp. 2281-2285).

6. Ha, Q. P., Negnivitsky, M. \& Man, Z. (1996). Sliding mode control with fuzzy tuning, IEEE Transaction on Fuzzy Systems, 67,78-86.

7. Hung, J. Y., Gao, W. \& Hung, J. C. (1993). Variable structure control: A survey, IEEE Transactions on Industrial Electronics, 40(1), 2-22. 
8. Janardhanan, S. \& Bandyopadhyay, B. (2005). A new algorithm for discrete-time sliding mode control using infrequent output observation, Asian Journal of Control, 7(3), 310-318.

9. Kumar, S. N. \& Kumar, C. S. (2010). Design and implementation of adaptive fuzzy controller for speed control of brushless DC motors, International Journal of Computer Applications, 1(27), 36-41.

10. Moldoveanu, F. (2014). Sliding mode controller design for robot manipulators, Bulletin of the Transilvania University of Braşov, 7(56), 97-104.

11. Pan, Y., Furuta, K., Suzuki, S. \& Hatakeyama, S. (2000). Design of variable structure controller. In Proceedings of the 39th IEEE Conference on Decision and Control (pp. 1685-1690).

12. Perruquetti, W. \& Barbot, J. P. (Eds.). 2002. Sliding mode control in engineering, Control engineering. M. Dekker, New York.

13. Sabanovic, A., Sabanovic, N. \& Onal, C. (2005). Sliding modes in motion control systems, Automatika, 46, 17-27.

14. Selisteanu, D., Petre, E. \& Rasvan, V. B. (2007). Sliding mode and adaptive sliding mode of a class of nonlinear bioprocesses, International Journal of Adaptive Control and Signal Processing, 21, 795-822.
15. Setiawan, T., Widodo, R. J., Mahayana, D. \& Pranoto, I. (1997). Design non-linear system with sliding mode control, IFAC Proceedings, 34(11), 190-197.

16. Srininvasan, K., Gandhi, D. \& Venugopal, M., (2014). Spacecraft attitude control using control moment gyro reconfiguration, Studies in Informatics and Control, 23(3), 285-296. doi: 10.24846/v23i3y201407

17. Temel, T. \& Ashrafiuon, H. (2012). Sliding mode control approach for fast tracking, Electronics Letters, 48(15), 916-917.

18. Utkin, V. I. (1993). Sliding mode control design principles and applications to electric drives, IEEE Transaction on Industrial Electronics, 40(1), 23-36.

19. Varghese, G. C., Fernandez, B. \& Hedrick, J. K. (1988). Stable, robust tracking by sliding mode control, Systems and Control Letters, 10(1), 27-34.

20. Wang, L. X. (1993). Stable adaptive fuzzy control of nonlinear systems, IEEE Transactions on Fuzzy Systems, 1(2), 146-155.

21. Young, D. K., Utkin, V. I. \& Zgune, U. O. (1999). A control engineer's guide to sliding mode control, IEEE Transactions on Control Systems Technology, 7(3), 328-341. 